\title{
An IP network-based signal control system for automatic block signal and its functional enhancement
}

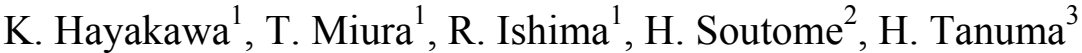 \\ \& Y. Yoshida ${ }^{4}$ \\ ${ }^{I}$ East Japan Railway Company, Japan \\ ${ }^{2}$ Hitachi Ltd., Japan \\ ${ }^{3}$ Toshiba Corporation, Japan \\ ${ }^{4}$ Daido Signal Ltd., Japan
}

\begin{abstract}
Conventional signalling systems for automatic block signals have several problems, such as the complicated wiring work for relays and unreliable simplex devices. We developed an IP Network-based Signal Control System for Automatic Block Signal. A Logic Controller (LC), placed in a signal house, integrates several control logics of signalling field devices. A Field Controller (FC), placed near each automatic block signal, electrically controls the signalling field devices. The LC and FCs exchange the control data through an IP network, the connection of which is simpler than conventional relay circuits. In order to enhance maintainability and transport stability in introducing this system into service, the following new functions have been developed. A diagnostic function enables the FCs to recognize where a malfunction of field devices or cables has happened, by means of current sensors added in the FCs. It is also possible to maintain train operations, even in the case of a malfunction of track circuits, by tracing train movements around the track circuits concerned. These new functions are expected to contribute to quick restoration at the breakdown in the signalling devices and the stable transport. We have carried out field tests to confirm their functionalities and characteristics in the wayside environment. This paper describes the above-mentioned functional enhancement and its field test results as well as an outline of an IP Network-based Signal Control System for Automatic Block Signal.
\end{abstract}


Keywords: IP-network, PON, automatic block signal, track circuit, current sensor, maintainability.

\section{Introduction}

Between stations we have many signalling devices such as block signals, track circuits and balises of ATS-P (Automatic Train Stop with Patterns, which is Automatic Train Protection). Each signal device is different in structure and has individual functionality. Those devices are connected with each other through relay contacts and signal cables to exchange status/control data (Figure 1 (a)). This system structure of conventional signalling systems has the following issues.

(1) Huge amount of signal cables and wiring work

(2) Complicated relay circuit logic

(3) Simplex system (no backup in case of equipment failures)

(4) Insufficient information on maintenance and failures from distant locations

To deal with (2), (3) and (4), we introduced a signalling system, of which all control units are duplex and placed in a signal house with good environment, to the Chuo line between Tokyo and Takao (the Chuo-line system Figure 1 (b)). The system, however, controls each signalling field device placed along the wayside directly from the central signal house through enormous number of metal cables, which require high cost and much construction work. The cables are still simplex, while all control units are duplex.

In order to cope with those issues, we started with the development of a NEtwork-based Signal Control System for Automatic Block Signals (NESCSABS) [1] on the basis of a signalling system that controls the field devices in a station yard through an IP network [2]. NESCS-ABS (Figure 1 (c)) aims at reducing cables and interface relays as well as improving the availability and maintainability of signalling systems.

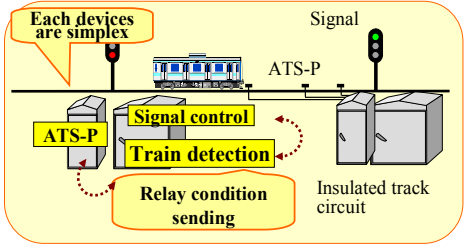

(a) Conventional Signalling System

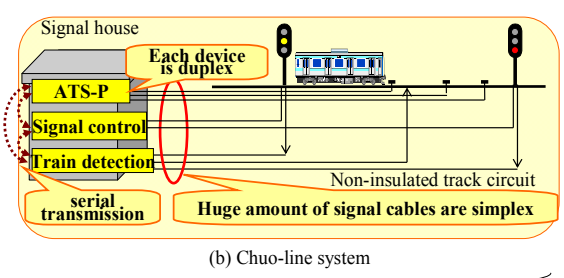

(b) Chuo-line system

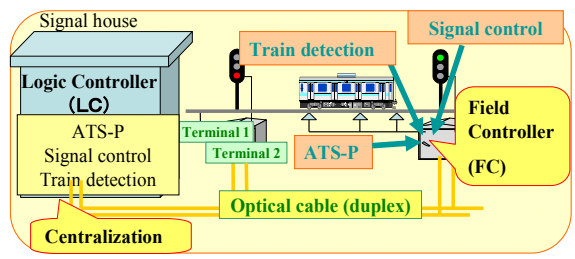

(c) Network-based signal control system for automatic block signals

Figure 1: Comparative system. 


\section{Overview of the NESCS-ABS}

Figure 2 illustrates the system configuration of the NESCS-ABS. The system mainly consists of a LC, FCs, an optical network and a remote monitoring system. Devices are connected with each other by an optical network, and data is transmittal by IP communication. The system including the optical cables has a duplex structure for reliability improvement.

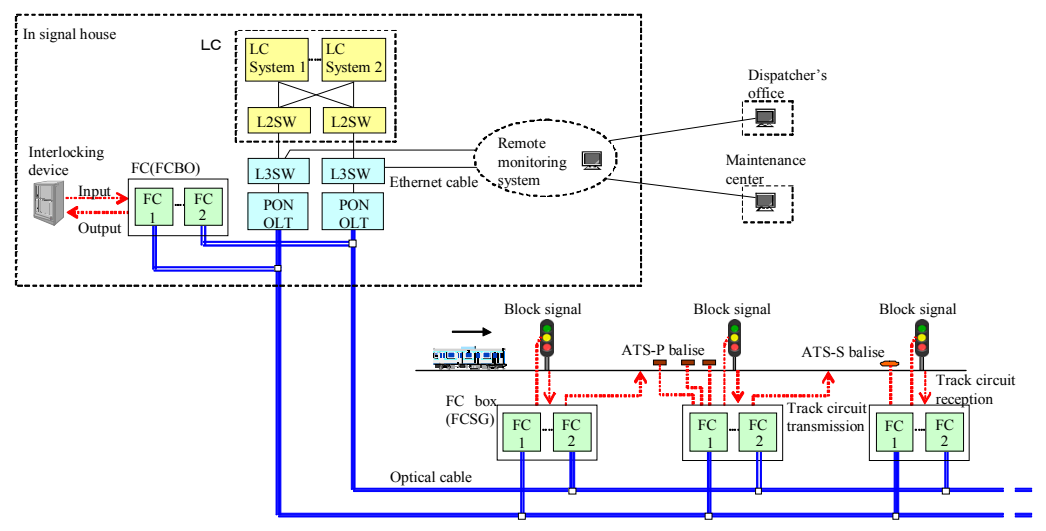

Figure 2: $\quad$ System configuration of the NESCS-ABS.

\subsection{LC}

LC is equipment that centralizes control logic of signalling devices for automatic block signals, and is placed in the signal house of a station. It determines the specific control of each signalling device on the basis of the information from FCs and the interlocking device, and it sends the command data to FCs via the optical network. It is built in a duplex structure with fail-safe control devices.

Main functions of the LC are as follows;

(1) Determination of occupancy of track circuits

(2) Determination of aspects of block signals, including repeat signals and distant route indicators

(3) Determination of the code information of ATS-P balises

Even in the case of a malfunction of track circuit, train operations are possibly by the newly-developed function for LC, which is discussed in 3.2.2.

\subsection{FC}

FCs are installed at each block section. The FC transforms command data from the LC into electric signals, and it controls signals, track circuits and ATS-P. They also transform the information of train detection by track circuits and other operation status of equipment along the tracks from electric signals into feedback data, and they transmit that to the LC via the optical network. 
As signalling devices to be controlled are concentrated around each block signal, the NESCS-ABS controls them by the FC which is installed for each signal block. FCs have a duplex structure with fail-safe control devices as LC do.

Figure 3 shows a connection of signalling devices to the FC. They are connected by metallic cables with connectors. It uses non-insulated track circuit to detect a train, and a transmitter and a receiver for that are also built in the FC.

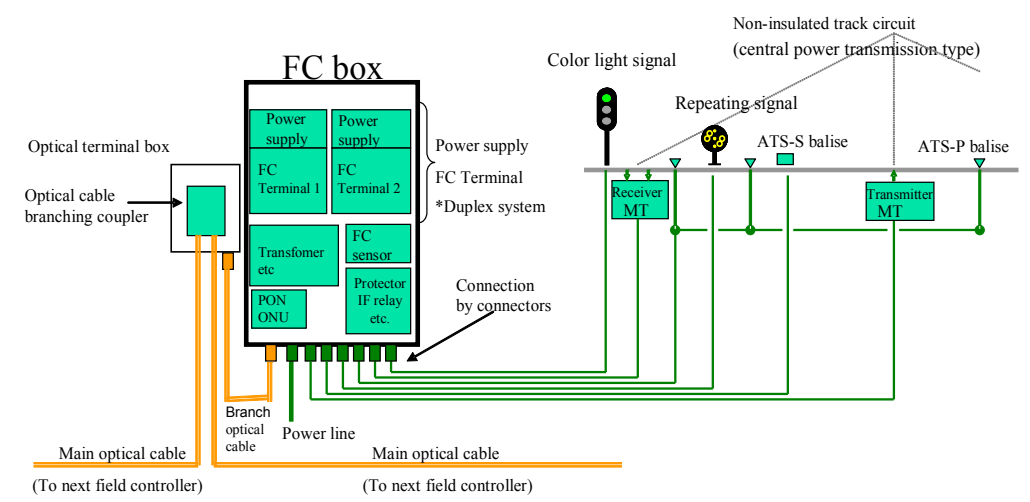

Figure 3: Connection of signalling devices to the FC.

\subsection{Optical network}

LC, FCs and other devices are connected via optical cables to reduce huge amount of cables. For the transmission method between LC and FCs, we have adopted the PON (Passive Optical Network) method that is a universal technology used for FTTH (Fibre To The Home) because PON is good in terms of ease of installation and maintainability and is well suited for laying along the tracks by the following reasons;

(1) With time-division multiplexing, PON can secure many lines to reduce the number of core wires.

(2) Optical cables of PON can be multi-branched by using couplers without power supply.

PON consists of an Optical Line Terminal (OLT) and Optical Network Unit (ONU). In our system, we set up the PON OLT in the signal house and place the PON ONU in the FC boxes along the tracks. Furthermore, individual network devices (PON OLT, L2SW and L3SW) and optical cables are multiplexed.

\subsection{Remote monitoring system}

The remote monitoring system (Figure 4) is a system to remotely obtain information on wayside signalling devices and to provide it at the dispatcher's office and the maintenance centre as detailed as in the signal house. It also enables resetting of devices for recovery from obstructions etc. at the dispatcher's office. The remote monitoring system consists of remote monitoring server, remote control server and remote monitoring terminals. 


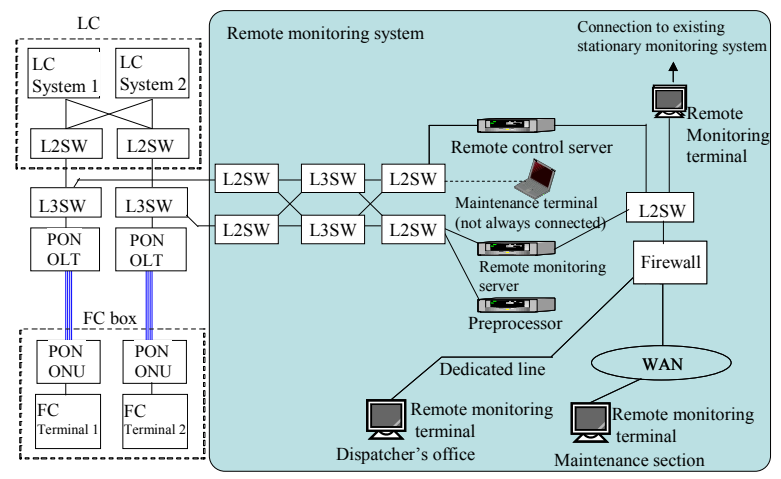

Figure 4: Remote monitoring system.

\section{Development of practical system}

In order to enhance maintainability and transport stability in introducing this system into service, we have improved and developed new hardware and functions on the basis of the issues found in the prototype system development.

\subsection{Development of hardware}

\subsubsection{Improvement of FC box}

We downsized the FC box taking into account the limited installation space along the tracks. But, as a result, problems remained in ease of installation at setup and maintainability at parts replacement due to internal parts being too closely packed.

Thus, we have enlarged the size of the box slightly and improved the component arrangement. Specifically, we have improved the arrangement to enable operation check and replacement of individual components from the front side of the FC and wiring from the rear side for better ease of installation and maintainability. And we have further modified the component structure of the FC to allow replacement for each internal unit.

Upon consideration of cases where a FC is installed on elevated sections and other places with heavy vibration, we have enhanced vibration isolation structure of the FC box. That lowers the vibration of the FC and other components to the specified level or less for vibration at $2 \mathrm{G}$ (double of the previous spec).

\subsubsection{Development of the FCBO (FC for system BOundary)}

In order to put this system into practical use, it has to have a serial interface with interlocking equipment and a transmission interface with ATS-P encoders, in addition to the relay interface, at the boundary with other systems. We have thus developed the FC for system BOundary (FCBO) that has those interfaces.

For example, the information required to control ATS-P over the system boundary is transmitted between the conventional ATS-P encoder and FCBO. 


\subsection{Development of block section extension in track circuit failures}

\subsubsection{Overview}

When a track circuit for automatic block signals is failures, we currently secure train operation by non-block operation with full attention.

But that limits the number of train services, seriously affecting stable transport. We have thus developed a function for extending a block section to maintain train operations without non-block operation with full attention by specifying the faulty track circuit.

In the development of the practical system, we have developed the following basic functions for that.

(1) Function to control occupancy or clear of the section with faulty the track circuit by tracing train movement around the track circuits concerned.

(2) Function to set the function in (1), above, by a remote monitoring terminal.

\subsubsection{Vision for the extension of block section}

When a track circuit unit of a FC is breakdown, the LC judges its track circuit as occupancy, and controls the stop aspect. If a dispatcher designates the faulty track circuit by remote monitoring terminal and the first train goes through that by the dispatcher's operation, the LC judges that as clear and controlled the

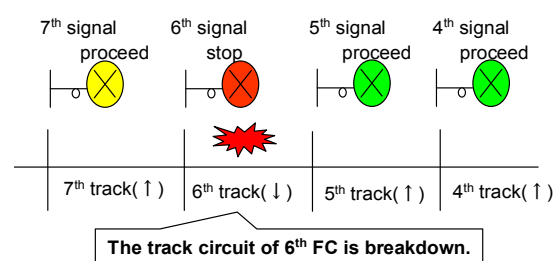

(a) The breakdown occurred.

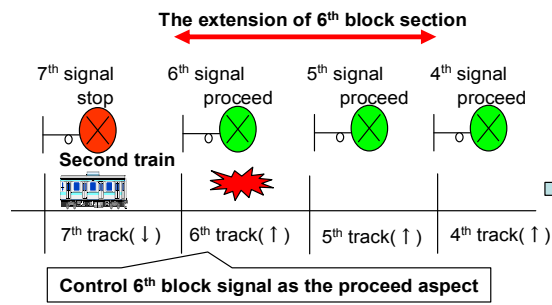

(c) The occupancy timing of $6^{\text {th }}$ track circuit

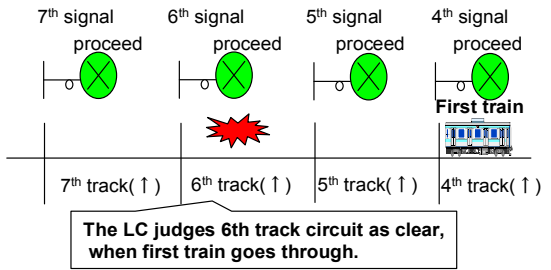

(b) The process of the extension of block section

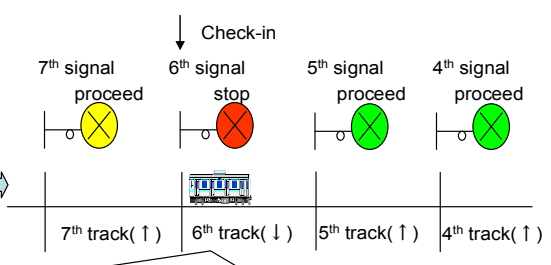

When $7^{\text {th }}$ track circuit is clear, $6^{\text {th }}$ track circuit is occupied.

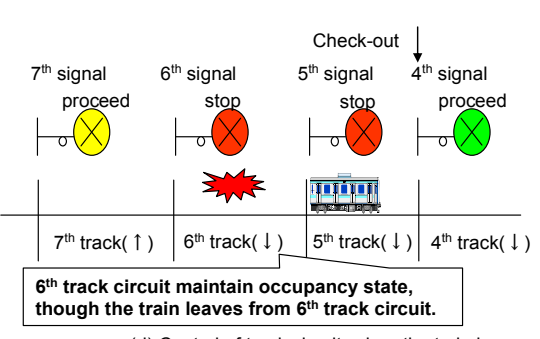

(d) Control of track circuit, when the train leaves

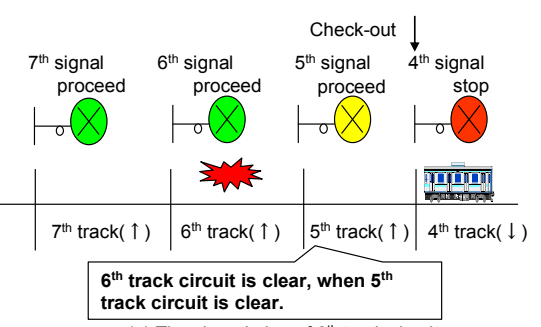

(e) The clear timing of $6^{\text {th }}$ track circuit

Figure 5: Vision for the extension of block section. 
block signal as the proceed aspect. After the second train, in order to detect the train on the faulty track circuit, the LC judges that state by tracing train movements around the track circuits concerned. When outside track circuit of the faulty track circuit changes from occupancy to clear, the LC judges the faulty track circuit as occupancy (check-in). Next, when inside track circuit of the faulty track circuit changes from occupancy to clear, the LC judges the faulty track circuit as clear (check-out). We illustrate an example of the movement on Figure 5.

(a) When the track circuit unit in 6th FC is breakdown, 6th track circuit is occupancy and 6th block signal is stop aspect. But, when a dispatcher checks no problem for the rail state and designates 6th track circuit by remote monitoring terminal.

(b) After first train goes through 6th and 7th track circuit by a dispatcher's operation, the LC judges 6th track circuit as clear and controls the 6th block signal as the proceed aspect.

(c) When 7th track circuit changes from occupancy to clear by tracing second train movements, the LC judges the faulty 6th track circuit as occupancy, and controls 6th block signal as stop aspect. The occupancy timing of 6th track circuit delay by comparing normal track circuit state. This is no problem; because we allow only one train to occupy one section, and the train can't proceed into the next section with occupancy.

(d) The train leaves from 6th track circuit and is only on the 5th track circuit, but 6th track circuit maintain occupancy state.

(e) When the train leaves from 5th track circuit and it changes from occupancy to clear, the LC judges 6th track circuit as clear and controls 6th block signal as proceed aspect. The clear timing of 6th track circuit delay by comparing normal track circuit state. This is no problem; because 6th block signal changes from stop aspect to proceed aspect after the train leaves from inside 5 th track circuit.

We describe that as check-in and check-out method by the extension of block section. As respects this function at a failure of the track circuit detector, we develop the basic control logic of the track circuit state at a failure. But in preparation for the practical operation, we need to decide the confirmation method of the setup procedure, the rail state and the presence of a train for the broken track circuit unit of a FC. Forward we need to develop the further function.

\subsection{Development of the practical system in the remote monitoring system}

\subsubsection{Function of identifying the faulty part in device failures}

When a device failure occurs once by conventional signal equipment, great time is needed for the identification and restoration, because a candidate for the faulty part covers a wide range. In order to control various devices by FCs of the NESCS-ABS, when a failure occurred to FCs and signalling devices, it's necessary to identify the faulty part promptly with pinpoint accuracy and be restored by a short time. So we improved to indicate a faulty part to remote monitoring terminal. The FC combines the plural sensor information and the 
judgment logic, and identifies the faulty part, the degradation of the device and the operation state of each unit in the FC. We describe the mechanism of a main sensor in the FC and specification of a faulty part.

\subsubsection{FC sensor}

A FC sensor checks a driving current (DC) of the output relay controlled by the FC and the temperature inside the FC box. As FCs consist of electronic devices in the FC box, it's necessary to cool by fans and heat sinks, so that the temperature in the FC box will be also below the fixation temperature at the time of hotness in the most heat time of year. When a FC sensor detects that the temperature inside the FC box is beyond the preset temperature, a FC sensor controls fans. Fans are diagnosed by comparing their control and actual operating states.

\subsubsection{Sensor for power source}

Power supply line systems along the tracks are duplicated, but there is no monitoring mechanism which system actually provides power with field devices. A sensor for power source performed the voltage monitoring at power supply lines of 2 ancestries and the output end and watched normal operation of power supply, as shown in Figure 6.

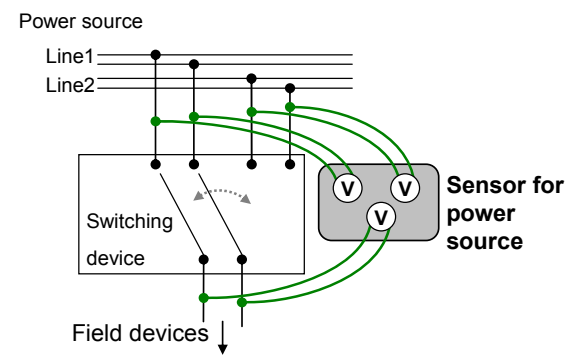

Figure 6: $\quad$ Sensor for power source.

\subsubsection{Current sensor of the signal lamp}

A signal lamp consists of a lot of LED balls, and when each LED balls break with the passing years, consumption current gradually reduces. On the basis of

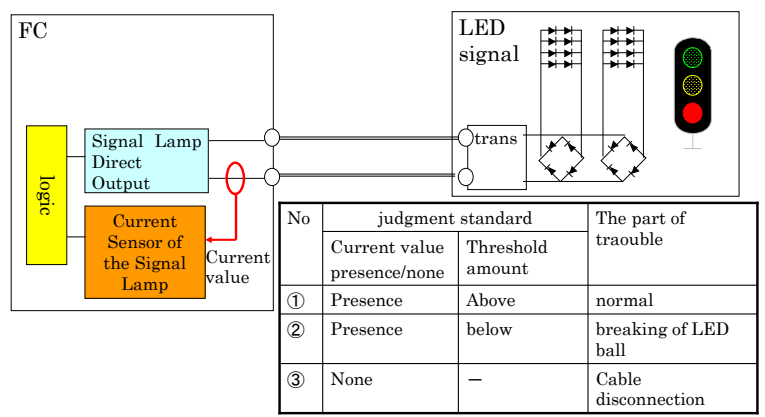

Figure 7: Current sensor of the signal lamp. 
this characteristic, the FC detects the LED problem when the current gets below a certain threshold amount, which enables to exchange the signal lamp before it completely breaks. When the current value is none, the FC decides on the cable disconnection between the FC and signal lamp. Thus a faulty part of the breaking of signal lamp or cable disconnection is identifiable, by combining a certain threshold amount for the current value with a presence of the current value (Figure 7).

\subsubsection{Sensor for track circuit}

The track circuit, which detects a train, consists of a sending unit and a receiving unit in the FC, rails and cables connecting them. The FC can detect its own problems by self-diagnosis, but when problems occurred on the outer side of the FC, it is not possible to specify where they actually occurred.

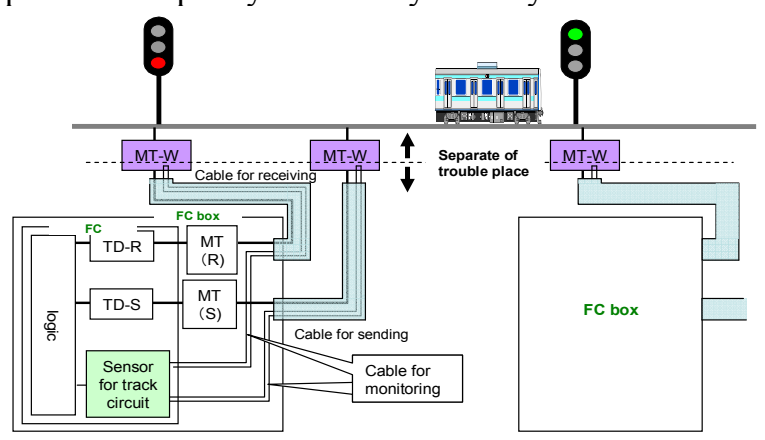

Figure 8: $\quad$ Sensor for track circuit.

To cope with this situation, a wayside transformer (MT), which is inserted at the end of rails, is made use of (see Figure 8). The FC can distinguish the problems of the cables from the problems beyond the MT (i.e. the problems of wayside connections, rails, etc.) by checking a signal obtained by an additional sensing coil to the MT.

The output level of the transmission unit is set appropriately so that the input level of the reception unit is not influenced by weather, by monitoring its input level.

\subsubsection{Sensor for ATS-P}

ATS-P is a train protection system, and train control data is transmitted from the grand to trains through balises. As shown in Figure 9, the transmission current from the FC to the ATS-P balises monitored. The FC distinguishes the problem parts by checking the monitoring results and the feedback data from the ATS-P balise. Figure 9 indicates its diagnostic criteria.

\subsubsection{Use of the monitoring data for the preventive maintenance}

The monitoring data of each device acquired by the NESCS-ABS is accumulated and the average, the greatest and the minimum value on the 1st are calculated in a remote monitoring server. These data is taken out from a remote monitoring server by need and the monitoring data of each device is displayed at the remote 


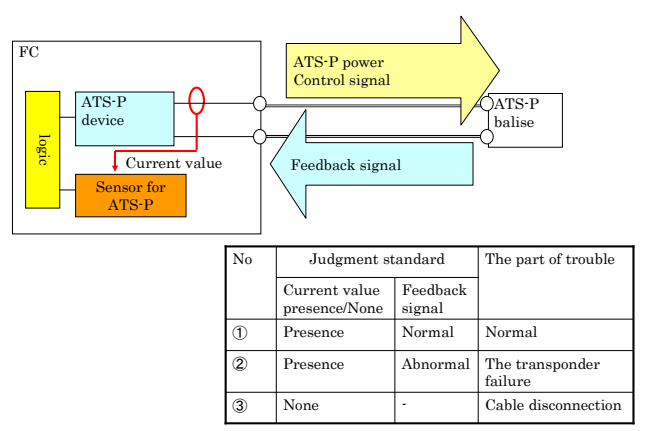

Figure 9: $\quad$ Sensor for ATS-P.

monitoring terminal by graph. The user acquires the tendency by the date of each device, and utilization to preventive maintenance is expected.

\section{Test of the practical system}

We have carried out stand-alone and combination functional tests of each device, field tests and operation environment checks of the NESCS-ABS.

\subsection{Field tests}

We have set up the developed practical system between Mabashi and KitaKashiwa stations near Kita-Kogane station on the Joban rapid line for the field tests. In those tests, we have evaluated the control performance, the transmission performance, and the reliability and environment resistance of the system in the long-term operation in the on-site environment. The field tests started in June 2008.

\subsubsection{System configuration}

Figure 10 illustrates the field test system configuration. The LC was installed in the former signal house of Kita-Kogane station. Six FCs (five FCSG and one FCIF) were installed in a total of five block sections: outbound No. 2 and No. 1 between Mabashi and Kita-Kogane and inbound No. 1, No. 2 and No. 3 between Kita-Kogane and Kita-Kashiwa. In order to input the aspect information of the entry signals with the existing interlocking device, we have installed two FCBOs as the interlocking equipment interface. The remote monitoring system is in the former signal house of Kita-Kogane station. We have built an optical network between those to control the FC and simulated signalling devices for automatic block signals (block signals, repeating signals, track circuits, ATS-P and distant route indicators). In the field tests, we are making comparisons of the operation between the practical system and the existing system.

In order to verify the validity of the system in the actual train operation in the field tests, the system imports the conditions of the existing devices at relay contact points to compare the control details and control timing of the existing devices and the network-based signal system. 


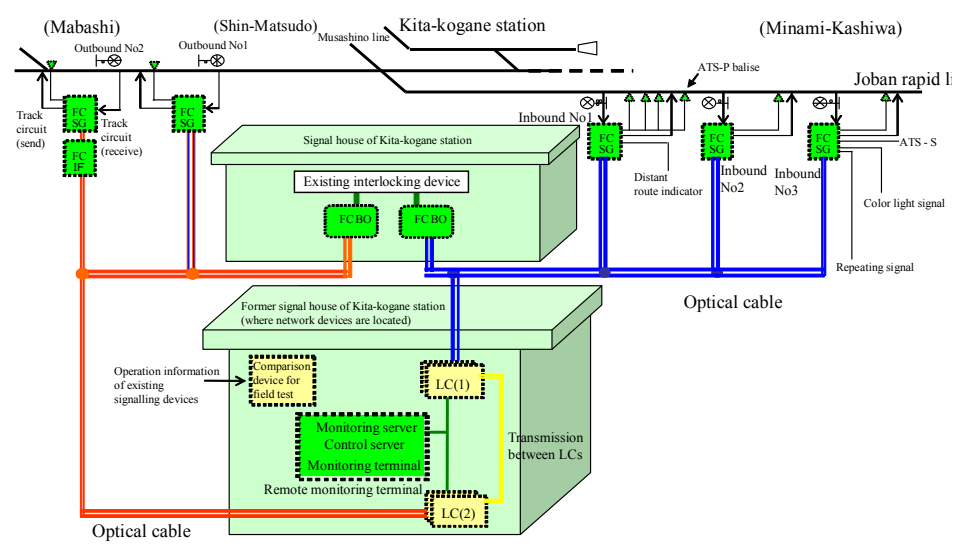

Figure 10: System configuration of the field test.

\subsubsection{Evaluation}

Evaluation items in the field tests are reliability and performance. The number of train runs in the field test section is approx. 200 a day each for inbound and outbound lines.

The evaluation criterion of reliability is whether or not any device failure occurs (if any, whether or not the cause and the countermeasure are specified). Failures occurred at some devices, but we found the causes and took countermeasures, after which the system has been operating normally. We have found no further failures and operation stops of field devices due to external environmental causes such as high temperature in summer and electromagnetic noise.

The evaluations of performance are as follows, proving that the required performance is met.

(1) Non-insulated track circuit: We checked the position of the boundary between adjacent track circuits, short-circuit sensitivity, train detection and other performance items, and found that the specified train detection performance is met.

(2) ATS-P: We confirmed that functions such as control code encoding and onboard code reception are equal to the existing ATS-P.

(3) Signal aspect control, output etc.: We confirmed that those correspond to existing control. In some cases those did not correspond, but the cause was the difference of the train detection timing. That occurred because this system used electronic track circuits while the existing system uses relay track circuits. We proved that there were no problems with the logic processing of the LC.

(4) Transmission: We measured no errors of networking devices.

(5) Remote monitoring server, remote monitoring terminal: We confirmed that those could detect device failures and output warnings. 


\subsection{Environment-resistant test}

As we install the FC using electronic devices along the tracks, we defined the minimum values of each environmental-resistance which the FC box must have, referring to IEC (International Electrotechnical Commission) and JIS (Japanese Industrial Standard) for conventional signal field devices. We designed the FC to cope with those conditions. Table 1 shows the main environmental conditions of the FC. Concerning the condition of EMC, we refer to IEC 62236-4, applicable to signalling and telecommunication apparatus which is installed in the railway environment. As for Impulse surge voltage, we defined $30 \mathrm{kV}$ for power port and $20 \mathrm{kV}$ for $\mathrm{I} / \mathrm{O}$ port. We performed the environment-resistant tests for the FC box based on the defined environmental conditions at testing sites. The FC has passed all the environment-resistant tests for the FC Box based on the defined environmental conditions at testing sites. The FC has passed all the environment-resistant tests and satisfied those environmental conditions.

Table 1: $\quad$ Main environmental-resistant of the FC.

\begin{tabular}{|c|c|}
\hline Internal temperature & -10 to $60{ }^{\circ} \mathrm{C}$ \\
\hline Vibration & 10 to $500 \mathrm{~Hz}$ (over 1G) by JIS E3014 type-2 \\
\hline Water-proof & JIS E3017 R2 \\
\hline $\begin{array}{c}\text { Electro Magnetic } \\
\text { Compatibility (EMC) }\end{array}$ & IEC 62236-1, 4 \\
\hline \multirow{2}{*}{ Impulse surge voltage } & $30 \mathrm{kV}$ for power port \\
\cline { 2 - 2 } & $20 \mathrm{kV}$ for I/O port \\
\hline
\end{tabular}

\section{Conclusion}

We have made improvements and addition of functions that would be helpful to more stable transport and higher maintainability and ease of installation. We are now working to determine the final specifications.

For the future, we plan to further preparations to put the system into practical use in the greater Tokyo area.

\section{References}

[1] R. Ishima, Y. Fukuta, M. Matsumoto, N. Shimizu, H. Soutome, M. Mori “A New Signalling System for Automatic Block Signal between Stations Controlling through an IP Network," WCRR, May.2008.

[2] Y. Hirano, Takashi. Kato, T. Kunifuji, T. Hattori, Tamotsu Kato, "Development of Railway Signalling System Based on Network Technology," IEEE SMC, Oct.2005. 